\section{PERSPECTIVAS \\ EN INTELIGENCIA \\ 2020}

\title{
Espionaje y normatividad colombiana, integridad del Estado Social de Derecho y la Seguridad Nacional
}

\author{
Espionage and Colombian regulations, integrity of the Social State \\ of Law and National Security
}

Sergio Andrés Ramírez Figueredo ${ }^{1}$

(1) Universidad de San Buenaventura, Bogotá - Colombia, sarfker@gmail.com

\section{Resumen}

Los riesgos del espionaje tradicional, donde ciudadanos extranjeros o nacionales efectúan contacto con agencias de seguridad o actores no estatales extranjeros, representan posibles caminos para la fuga de información del Estado, así como una vulnerabilidad ante potenciales daños a efectuarse en el territorio nacional, sea contra activos estratégicos, ciudadanos nacionales o intereses foráneos presentes en Colombia.

Las medidas normativas en Colombia, consignadas en el Código Penal, Ley 599 del 2000, estipulan la aprehensión de todo individuo o grupo de estos, detenido y juzgado por comprometer secretos de carácter vital para la seguridad del Estado. La arquitectura del Estado Social de Derecho, definida por la Constitución Política de 1991, exige la primacía de los parámetros de derechos humanos y DIH, como principio de legalidad que se extiende a casos de espionaje ejecutados por connacionales y extranjeros.

La prevención ante el ingreso o eventual trámite de expulsión a ciudadanos extranjeros acusados por acciones de espionaje son potestad de Migración Colombia; los cuerpos de inteligencia y contrainteligencia nacionales ejercen funciones de rastreo, prevención y monitoreo de eventuales focos de infiltración de actores externos o internos, pero el manejo último tanto de la situación de permanencia o status jurídico de los ciudadanos extranjeros acusados por espionaje son ámbito de la agencia de Migración y del sistema judicial encargado de definir la acción penal.

En consecuencia, el objetivo de investigación apunta a observar cómo coexisten la esfera garantista del DIH y los compromisos de Seguridad Nacional ante el fenómeno del espionaje en Colombia; ante lo cual como hipótesis se considera que el carácter disperso del contexto normativo encargado de tipificar tales actividades, afecta la aplicabilidad de medidas penales, de tal forma que el camino resolutorio es la activación de protocolos de expulsión del individuo o grupos de extranjeros relacionados con casos de espionaje.

Palabras clave: Espía; Derecho Internacional Humanitario; Seguridad Nacional; Estado Social de Derecho; Migración Colombia; Inteligencia y Contrainteligencia. 


\section{Abstract}

The risks of traditional espionage, where foreign or national citizens make contact with security agencies or foreign non-state actors, represent possible paths for the leakage of information from the State, as well as a vulnerability to potential damage to be carried out in the national territory, whether against strategic assets, national citizens or foreign interests present in Colombia.

The normative measures in Colombia, established in the Penal Code-Law 599 of 2000, stipulate the apprehension of any individual or group of these, detained and tried for compromising secrets of a vital nature for the security of the State. The architecture of the Social State of Law, defined by the Political Constitution of 1991, demands the primacy of the parameters of human rights and IHL, as a principle of legality that extends to cases of espionage carried out by nationals and foreigners.

The prevention of the entry or eventual expulsion procedure of foreign citizens accused of espionage actions, are the power of Migración Colombia; The national intelligence and counterintelligence bodies perform functions of tracking, preventing and monitoring eventual foci of infiltration by external or internal actors, but the ultimate management of both the permanence or legal status of foreign citizens accused of espionage, are the scope of the aforementioned Migration Agency, and the judicial system in charge of defining criminal action.

Consequently, the research objective aims to observe how the guarantee sphere of IHL and National Security commitments coexist in the face of the phenomenon of espionage in Colombia; Given which, as a hypothesis, it is considered that the dispersed nature of the normative context in charge of classifying such activities affects the applicability of criminal measures, before which the resolutive path is the activation of expulsion protocols for the individual or groups of foreigners related to cases of espionage.

Keywords: Spy; International Humanitarian Law; National Security; Social State of Law; Colombian Migration; Intelligence and Counterintelligence.

\section{Introducción}

La discusión sobre el tratamiento de casos de espionaje en el ordenamiento jurídico e institucional colombiano es un espacio de análisis con limitada producción académica, en relación a la pertinencia y relevancia que actualmente ostentan asuntos vinculados con tal ámbito. Muestra de esto son los casos referenciales como aquel protagonizado por el ciudadano venezolano Carlos Manuel Pino García, expulsado por Migración Colombia en diciembre de 2018, al representar su permanencia un riesgo para la Seguridad Nacional (El Tiempo, 2018). Casos de similar índole tuvieron como actores centrales a otros ciudadanos extranjeros, como el cubano Raúl Gutiérrez Sánchez, expulsado el 23 de mayo de 2019, después de que el juez quinto penal de Bogotá estimara que las publicaciones virtuales de Gutiérrez no representasen una condición material de amenaza contra el país (Collazos, 2019).

Los riesgos del espionaje en la actualidad extienden su realización más allá de las labores efectuadas por sujetos al servicio de actores regionales latinoamericanos, 
muestra de ello es el caso de espionaje registrado por parte de dos funcionarios diplomáticos rusos, expulsados a finales del año 2020 por parte de las autoridades colombianas, en el marco de la solicitud efectuada por la Cancillería colombiana ante la información suministrada por la Dirección Nacional de Inteligencia DNI (Colombian Risk Analisys, 2021), sobre las actividades sospechosas de dichos extranjeros, en cuanto efectuaban desplazamientos y acciones poco usuales dentro de los roles y ámbitos de desenvolvimiento tradicionales para funcionarios diplomáticos.

A la luz de dichos acontecimientos, se hace necesario considerar los elementos normativos vigentes respecto al espionaje, y su articulación, retomando los principios del Derecho Internacional Humanitario que rigen al país a través del bloque de constitucionalidad estipulado en la Constitución Política de 1991. A partir de esto último, instituciones como los organismos de inteligencia o Migración Colombia supeditan sus principios organizativos (Ley 1621/13 y Decreto 4062 de 2011 respectivamente) al cumplimiento de parámetros legales de derechos y deberes fundamentales propios de un Estado Social de Derecho. Por su parte, los compromisos constitucionales del Estado ante sus connacionales exigen la protección en términos de integridad física y preservación del entorno viable para el desarrollo de los proyectos de realización de vida individuales o colectivos, destacándose la figura de la Seguridad Nacional como elemento que integra la atención de toda clase de riesgos y amenazas contra el Estado y sus habitantes, entre los cuales se adhieren las actividades de espionaje.

Los elementos recabados en el marco normativo anexo a esta publicación permiten identificar cómo las figuras jurídicas que tipifican el suceso delictivo del espionaje afrontan notorias limitaciones en cuanto proyectar fuerza penal de carácter disuasorio ante la comisión de dichas actividades, así como impactan en dispersar los parámetros de delitos a castigar, derivando aquello en dificultades y contradicciones interpretativas de los administradores de justicia, de cara al análisis, emisión de concepto jurídico y aplicación del marco penal establecido para el castigo de las actividades asociadas a los delitos de espionaje.

\section{Los derechos fundamentales y la organización del Estatuto legal de inteligencia y contrainteligencia}

Al dar inicio a este artículo, se establece como objetivo identificar cómo interactúa la estructura legal colombiana vinculada con el ámbito de Seguridad Nacional -y en especial respecto al componente del espionaje-, ante la composición normativa nacional enmarcada dentro del seguimiento y amparo de los derechos fundamentales y el respectivo respeto de las actividades del Estado ante la potencial vulneración de aquellos. Entre el equilibrio de esas dos esferas, resalta de por medio la persistencia de múltiples aspectos de amenaza y/o riesgo para la integridad de la nación. Como primer paso hacia dicho objetivo, se hace necesario caracterizar el espíritu normativo que da cabida a reglamentaciones como la Ley estatutaria 1621 de 2013 de Inteligencia y Contrainteligencia, cuyo marco de responsabilidades dentro del Estado Social de Derecho estipula la primacía de las garantías sociales, reconocimiento y garantía de los derechos fundamentales desarrollados en los textos constitucionales a través de la figura de una ley estatutaria (Jiménez, y otros, 2016). 
Respecto al Estado Social de Derecho y su sustrato normativo estipulado en el Artículo Primero de la Constitución Política de 1991, se puede observar la dimensión del alcance del efecto declarativo de la Carta Magna; desde la apreciación teórica del concepto destacado por Gilberto Tobón Sanín (2018), se hace referencia a principios como la intervención del Estado en los que este debe actuar de forma activa a manera de gestiones de gasto social, definidas por el autor como el fomento dispuesto para garantizar la armonía social, reflejada en agendas como la prestación de servicios públicos, salud, vivienda, seguridad social y, en consecuencia, derechos económicos y sociales.

Sobre esas bases partió la discusión de la validez constitucional del entonces proyecto de ley encargado de conformar la vigente Ley de Inteligencia y Contrainteligencia, y analizado en su momento desde la Sentencia C-540 de 2012, en la que se asumió una consecuente lectura sobre los alcances y armonización del revisado cuerpo legal, en aras a que este no implicase la violación de los derechos fundamentales para su satisfactoria aprobación, de tal forma que la arquitectura de la Ley 1621/13 de carácter estatutario se integra bajo la disposición del Artículo 152 de la Constitución Política de Colombia, en cuanto asume el tratamiento de asuntos relativos a los derechos y deberes fundamentales de las personas (Inciso A), así como los respectivos procedimientos y recursos para el resguardo de tales aspectos. La condición declarativa de las leyes estatutarias, como protectoras de las personas, implica que las garantías básicas de derechos y deberes se apliquen sin distinción de nacionalidad en el territorio colombiano, pero acatando la orientación normativa expuesta en el Artículo 100 de la Constitución Política, en lo referente a los extranjeros.

La providencia del Artículo 153 ratifica, por su parte, el papel obligatorio de revisión previa de todo proyecto estatutario de ley, a efectuarse por la Corte Constitucional, con el fin de que esta estudie y emita conceptos de exequibilidad o no, en relación al acatamiento de la máxima de amparo de derechos que consagra al Estado colombiano desde su Carta política. La exposición de apertura de la Sentencia C-540/12, sobre la antesala formativa de la Ley 1621/13, asoció en su momento la discusión de derechos fundamentales como a la intimidad, habeas data, el buen nombre, la honra, el acceso a la información, el debido proceso y el principio de legalidad, como criterios que obligaron a surtir el trámite más riguroso y elevado consignado en la Carta Magna para la eventual aprobación y entrada en vigencia de la Ley 1621/13, ante lo cual las labores a cumplir por la Inteligencia y Contrainteligencia han de asumir dichos parámetros de cumplimiento en el actuar de sus competencias.

Por su parte, en aras de contemplar el espectro normativo más próximo al referente temático del espionaje como amenaza a la Seguridad Nacional, se hace fundamental observar un mecanismo de interpretación legal del ordenamiento jurídico colombiano. Es así como tal perspectiva implica asumir el rol de aquellas consideraciones que, sin estar escritas en la Constitución de 1991, logran ser parte del entramado normativo del país. Sobre esa situación, Mónica Arango Olaya (2004) destaca la función del bloque de constitucionalidad, en razón a una figura que legitima el valor de los tratados y convenios internacionales suscritos por el Congreso y ratificados por el presidente de la República, cuestiones validadas en los artículos 9, 53, 93, 94, 102 y 214 de la Carta Magna. 
A partir del mencionado bloque, toma sustento y aplicabilidad el DIH en el contexto nacional. Una investigación de doctorado inédita, realizada en la Pontificia Universidad Javeriana (2002), resaltó cómo incluso la jurisprudencia constitucional ha debatido sobre las variables incidentes en la aplicabilidad de tales principios, llegando a concluir que se pueden adoptar de forma automática los principios del DIH, sin requerimiento previo de aprobación por parte del Congreso de la República, tal y como lo demanda el Art. 224 de la Constitución de 1991. De tal manera, los principios normativos sobre el espionaje aplican de forma implícita los referentes de jurisprudencia internacional, donde se plasman aspectos como el Reglamento relativo a las leyes y costumbres de la guerra terrestre o al Estatuto del combatiente y del prisionero de guerra.

En realidad, los anteriores referentes establecidos por el Comité Internacional de la Cruz Roja como principios del DIH revisten otra revisión especial por parte de la Corte Constitucional en la Sentencia C-225 de 1995, con el fin de esclarecer el carácter imperativo de vigencia de tales efectos internacionales en el escenario doméstico. En efecto, dicha Sentencia hace mención sobre los convenios de La Haya de 1889 y 1907 (regulación de la conducción de las hostilidades y el combate, entiéndase reglamento de la guerra terrestre) y el Derecho de Ginebra-DIH de la protección de los no combatientes en medio de las hostilidades, destacando que ante una interpretación jurídica en el ordenamiento nacional, que asociaba únicamente el DIH al Derecho de Ginebra, se cometían observaciones poco lógicas, ante lo cual la Corte Constitucional declara que dentro de los fines de resguardo del DIH, es vital considerar la relevancia de los principios proferidos por los convenios de La Haya y su regulación sobre los medios legítimos del combate, como fuentes del DIH.

De esta manera, ambos (convenios y Derecho) tienen igualdad de condición amparable al DIH según la interpretación de la Corte, en lo que se llama un entendimiento amplio de la cuestión. Por otra parte, la Sentencia C-225/95 analizó el margen de aplicabilidad del DIH en medio de conflictos armados no internacionales, y enfatizó en que su vigencia en el marco interno se da en todo caso sin excepción. Años después, esa condición de operatividad del DIH para una confrontación interna, fue revalidada por la Ley 1448 de 2011 o Ley de víctimas, en la que el Estado colombiano reafirma el reconocimiento sobre la existencia del conflicto armado interno, en tanto una facción de la población se ha alzado en armas en contra de la institucionalidad, con el fin de alcanzar el poder.

De tal manera, la apreciación sobre el conjunto de derechos fundamentales expuestos tanto por la legislación colombiana, como mediante los compromisos internacionales adquiridos por el país mediante el bloque de constitucionalidad, implican consecuentes impactos sobre el abordaje de los lineamientos normativos aplicables ante cuestiones de impacto en los parámetros de Seguridad Nacional, como lo son acá los asuntos relativos al espionaje. Las apreciaciones presentadas respaldan la validez por vincular la mención del Capítulo II del Derecho Internacional, relativo a la conducción de las hostilidades-CICR (actualizado en 2005), en el que se tipifica la condición de espía. En efecto, ese amparo legal ratifica el principio del debido proceso en rechazo a pena alguna que se realice sobre un espía sin un juicio previo. Su condición se expone así: 


\section{De los espías}

Art. 29. No puede considerarse como espía sino al individuo que obrando clandestinamente o con falsos pretextos recoja o trate de recoger informes en la zona de operaciones de un beligerante, con la intención de comunicarlos al enemigo [...]

A su vez, el Estatuto del combatiente y de prisionero de guerra (1989) adhiere: "Estatuto de prisionero de guerra: En caso de captura, tienen estatuto de prisionero de guerra: -los combatientes (C. III, art. 4; P. I, art. 44), excepto: -el espía cogido en flagrante delito (P. I, art. 46)..." y adicionalmente menciona: "el espía cogido en flagrante delito de espionaje no tiene derecho, incluso si es miembro de las fuerzas armadas al estatuto de combatiente (ni de prisionero de guerra)". Sumado a ello, se encuentran las puntualidades del IV Convenio de Ginebra (1949), que una vez más recalcan sobre el respeto hacia la integridad humana del individuo capturado bajo cargos de espionaje:
Artículo 5
$\mathrm{Si}$, en el territorio de una Parte en conflicto, esta tiene serias razones para considerar que una persona protegida por el presente Convenio resulta fundadamente sospechosa de dedicarse a actividades perjudiciales para la seguridad del Estado, o si se demuestra que se dedica, de hecho, a dichas actividades, tal persona no podrá ampararse en los derechos y privilegios conferidos por el presente Convenio que, de aplicarse en su favor, podrían causar perjuicio a la seguridad del Estado.
$\mathrm{Si}$, en un territorio ocupado, una persona protegida por el Convenio es capturada por espía o saboteadora, [...] dicha persona podrá quedar privada de los derechos de comunicación [...].

Ciertamente, partiendo de los elementos del Artículo 29 de DI, relativo a la conducción de las hostilidades-CICR, se manifiesta el notorio rezago de su apreciación ante el tema del espionaje, máxime cuando la mencionada normatividad internacional ha tenido su última actualización en 2005. Adicionalmente, la arquitectura de ese componente legal está diseñada para ser interpretada durante contextos de enfrentamiento bélico contra un actor simétrico (estatal), arrojando esto evidentes porosidades y relatividad en torno a la definición de responsabilidades o condiciones a ejercerse ante los espías en tiempos de paz.

La utilidad de esas apreciaciones se enlaza precisamente con los principios del DIH, algo que especialmente refleja el Artículo 5 del IV Convenio de 1949, en cuanto mantener las garantías de integridad física y jurídicas al individuo sindicado por espionaje, condición que efectivamente va más allá de si esos actos se presentan en contextos de enfrentamiento bélico, internacional o no. Es así como las garantías de respeto por los derechos fundamentales respecto al tratamiento de los espías representan el principal elemento articulador para la eventual conducción jurídica de los casos acontecidos en el país; claramente, por su parte, las instituciones de seguridad del Estado han de apegarse a tales principios normativos y desde allí han de operar ante la gravitante amenaza y riesgo del espionaje. 


\section{Acercamiento al rol de la contrainteligencia y la protección del interés nacional}

Ciertamente, es pertinente plantear una profundización sobre la ramificación de la contrainteligencia, tomando en cuenta su rol principal en la identificación de los riesgos y amenazas internos y externos que comprometen la Seguridad Nacional a raíz de acciones que pretendan extraer información relevante de alto valor, que atenta contra la estabilidad del Estado. Entre tanto, sobre su aplicación normativa mediante la Ley 1621 de 2013, se destaca su perfil de orientación y vigencia dentro de los parámetros del Estado Social de Derecho, tal y como se puede reflejar a continuación:

Artículo 2. Definición de la función de inteligencia y contrainteligencia. La función de inteligencia y contrainteligencia es aquella que desarrollan los organismos especializados del Estado del orden nacional, utilizando medios humanos o técnicos para la recolección, procesamiento, análisis y difusión de información, con el objetivo de proteger los derechos humanos, prevenir y combatir amenazas internas o externas contra la vigencia del régimen democrático, el régimen constitucional y legal, la seguridad y la defensa nacional, y cumplir los demás fines enunciados en esta Ley.

En ese Artículo, el rol de la contrainteligencia (y la inteligencia), como instrumento para garantizar la protección de la seguridad nacional, recalca asumir toda misión que suponga una amenaza o riesgo para ese fin del Estado; de esa forma, la declaratoria normativa entiende en su forma la inclusión de una diversa gama de actividades criminales e ilegales que contravengan a ese interés conjunto para la nación, de tal manera que el espionaje hace parte de los factores que el ejercicio de ambas especialidades depara relevancia permanente. Y en paralelo, se asienta el espíritu normativo afín al estatus legal como texto Estatutario, desde el cual se ha de garantizar el resguardo efectivo de compendios legales que puedan afectar los derechos fundamentales de los ciudadanos.

De otra parte, el Artículo 4, sobre los límites y fines de la función de inteligencia y contrainteligencia, antepone cualquier labor que de allí surja, al cumplimiento de los derechos humanos y al DIH, con lo cual la identificación de un elemento de amenaza contra la integridad del Estado, como lo es el espionaje, está supeditado al tratamiento respetuoso de los derechos fundamentales del individuo acusado por actividades de tal índole. Es entonces como, entre las características del Estado Social de Derecho y el resguardo de los intereses de Seguridad Nacional, se amplifica el sentido declarativo por asumir el bienestar de la sociedad colombiana, entre las garantías al trato justo para aquellos sindicados por actividades contrarias al mantenimiento de la seguridad del país, bajo el marco del principio de legalidad que ampara a la ciudadanía y, por otra parte, el deber del Estado en garantizar su seguridad e integridad para el desarrollo de los proyectos de vida de los sujetos y las garantías ampliadas para el conjunto social.

En medio de esas particularidades se gesta el marco de organización normativa del Estado colombiano en materia de inteligencia y contrainteligencia, en conjunta integración con los principios conceptuales que dan razón y justifican la existencia de esa clase de mecanismos en un Estado moderno. Según Mark Lowenthal (2000), 
la contrainteligencia cumple con todas aquellas labores para resguardar el valor de la inteligencia propia y sus operaciones, ante amenazas externas que pretendan alterar o sustraer información de alto valor; de esa primera impresión podría asumirse que la contrainteligencia cumple exclusivamente con labores anticipativas a la iniciativa del bando adversario, pero Lowenthal (2000) reafirma que sí existe una vocación ofensiva, en la medida en que se pueden preparar operaciones de contacto con "agentes enemigos" los cuales, de forma consciente o no, se encargarán de transmitir información falsa, previamente calculada, con el fin de desviar las intenciones del adversario; además de ello, el rol de la recolección de información también constituye un activo esencial en la contrainteligencia, en la medida en que se indaguen las capacidades y habilidades del enemigo, que representen una amenaza de extracción de información.

En términos generales, la inteligencia y la contrainteligencia comparten elementos de trabajo que implican un imperativo por sostener actividades mancomunadas que, a la postre, se manifiestan en los principios de seguridad del país. De tal forma que la contrainteligencia se encarga de actuar ante la gestión por resguardar los secretos vitales de carácter militar, tecnológico y estratégicos del Estado, en relación con los intentos activos o pasivos de la inteligencia de otros actores internos o externos, con los cuales la arquitectura de Seguridad Nacional pueda verse alterada o comprometida.

Y sobre tal necesidad esencial para la seguridad del Estado, se empareja en términos normativos y de cumplimiento de principios vinculantes internacionales, en cómo sostener una conducción respetuosa de los derechos humanos y la dignidad de los sujetos, ante eventualidades de alta susceptibilidad al tratamiento humano, como lo puede representar la detención de un agente espía que represente algún grado de amenaza y/o riesgo para los fines supremos de seguridad Estatal.

\section{Migración Colombia y el seguimiento al espionaje}

En el marco de disposiciones institucionales establecidas por el Estado colombiano para prevenir y efectuar acciones legítimas, ante la presencia de ciudadanos extranjeros que efectúen actividades no gratas en territorio nacional, y que pongan en riesgo la integridad del territorio y sus habitantes, se concibe la existencia y competencias de Migración Colombia para tal finalidad. Esa institución, de carácter civil del Estado colombiano, facultada por el Decreto 4062 de 2011 para realizar las labores de vigilancia y control del flujo migratorio, ha emergido como instrumento especializado que integró las funciones mencionadas, y administradas anteriormente por el extinto Departamento Administrativo de Seguridad-DAS. Como tal, Migración Colombia asume funciones de Policía Judicial, como lo consagra el Artículo $4^{\circ}$, Numeral 4, del Decreto 4062/11, que desde la observación conceptual del término, planteada por Luis Navajas Ramos (1999), constituye un cuerpo científico y especializado de carácter técnico, encaminado a determinar las actividades delictivas asociadas al área funcional en la que se desempeñe esa unidad administrativa. De tal forma, el Decreto 4062/11 también enfatiza sobre la gestión articulada con la Fiscalía General de la Nación, en concordancia con la apreciación de Navajas Ramos (1999) sobre la importancia de una estricta dependencia de la Policía Judicial a un ente normativo-penal que lidera, estudia y emite sentencias sobre los procesos de lucha contra el crimen.

Es entonces como Migración Colombia funge como mecanismo dispuesto para la 
protección de la soberanía; por ende, asume acciones en torno a la Seguridad Nacional y la protección de los connacionales ante riesgos o amenazas internas o externas que mediante la violación de las normativas migratorias alteren la integridad de la interacción económica, social, política y de otros tantos aspectos englobados en la vocación de cooperación internacional de Colombia. Es así como el fenómeno del espionaje representa una de las categorías a monitorear por parte del cuerpo técnico especializado de Migración Colombia, condición reflejada a través de las facultades para determinar la congelación del visado nacional y expulsión de algún ciudadano extranjero que represente algún tipo de peligro para la seguridad del país.

Migración Colombia, como organismo con funciones de Policía Judicial, revalida la importancia, deber y sentido de articulación para el intercambio de información, como lo señala el Artículo 27 del Decreto 4062/11, en lo que se interpreta como esfuerzos de carácter interagencial e interinstitucional planteados para la eficacia de los objetivos institucionales deseables, en donde la menor cuota de rivalidad e interferencia en el cumplimiento de los deberes se materialice en el cumplimiento de las metas institucionales en relación con las funciones y competencias de quienes intercambian información.

En concreto, la gestión pública sobre aspectos migratorios exige reciprocidad, máxime cuando una de las potenciales amenazas a las cuales se hace frente involucra una inminente interferencia o daño de origen externo o interno que exponga información de alto valor estratégico para el país. Por lo tanto, la esencia del Decreto 1067 de 2015 da cuenta de un principio de Seguridad Nacional, al destacar la importancia de gestionar procesos de información originaria de organismos de seguridad o inteligencia nacionales o externos, en los cuales se pueda soportar la determinación de Migración Colombia al rechazar o expulsar a individuos extranjeros del territorio nacional, en alusión a factores o comportamientos que estos lleguen a representar como potenciales vectores de riesgo y amenaza para la seguridad interna y orden público.

En consecuencia, se observa con lo anterior que el perfil de abordaje, que en última instancia se encarga de tomar acción concreta en casos de espionaje, involucra la competencia y determinación de ejecutoria a tomar desde Migración Colombia. Tanto el perfil civil de tal institución, como los protocolos de conducción de posibles casos de espionaje detectados en territorio colombiano, exponen entre líneas el perfil de acción reafirmado a partir del tipo de medidas implementadas, todas ellas amparadas dentro de los principios del DIH.

\section{El Código Penal y casuística aplicada}

Entre las herramientas normativas colombianas analizadas, aquella que dispone un grado de definición sobre el espionaje es el Artículo 463 del Código Penal, concibiendo el delito como elemento de riesgo o amenaza delictiva para la seguridad del Estado; como también lo relacionan la Ley 1621/13, o los decretos 4062/11 o 1067/15. Es así como en términos punitivos, el Código Penal colombiano o Ley 599 de 2000 detalla los términos de aprehensión de algún individuo condenado por actos de espionaje, en lo referente a comprometer los secretos de orden militar, económico o político del país, y que sean manipulados con fines degenerativos para los intereses nacionales; asimismo, enfatiza en adherir ese delito al pliego de actividades excluidas de beneficios sustitutivos del pago de la condena en una institución carcelaria. 
No obstante, en la práctica los casos vinculados con espionaje analizados, se identifica cómo persiste una oscilación de aplicación normativa, existente entre medidas de expulsión y acción discrecional por parte de Migración Colombia (tal y como lo señala la Resolución 6045/17 del Ministerio de Relaciones Exteriores) y la aplicabilidad normativa del Artículo 463 del Código Penal (de acción carcelaria ante hechos asociados al espionaje); de ese espectro dual, es el primer elemento de aplicación normativa el que muestra ser la línea de acción predilecta ante los casos de espionaje detectados en territorio nacional.

Un informe periodístico del diario El Tiempo (2019) reúne casos de ese tipo, destacándose en primer lugar el de un "pescador venezolano" detenido en el margen limítrofe entre el departamento de Vichada y Venezuela, en lo que posteriormente las autoridades colombianas reportaron como caso de espionaje, según la confesión del que en realidad era capitán de la Guardia Nacional Bolivariana. Paso seguido se expone cómo las autoridades activarían el protocolo de expulsión -en lo que a todas luces estipula el Decreto 1067/15 para la Unidad Administrativa Especial de Migración Colombia-; no obstante, ante el hallazgo de material probatorio sobre las actividades de espionaje, el individuo fue conducido por la Sijin de la Fiscalía para su judicialización, acorde con el Art. 463 del Código Penal.

Por su parte, el diario El Tiempo (2019) da cuenta de acontecimientos como el del ciudadano cubano Juan Manuel Peña García, expulsado el 16 de marzo de 2019 a causa de una serie de sospechosas acciones realizadas en cercanías a la base aérea de Palanquero (Caldas), sumado al hecho de registrar vínculos con el G2 cubano, según información recopilada por ese diario. Mientras que para el 17 de marzo, otro caso de presencia sospechosa en torno a una instalación militar de la Fuerza Aérea ubicada en Funza-Cundinamarca, dio cuenta del ciudadano venezolano Brayan Andrés Díaz Díaz, el cual fue puesto en custodia ante las autoridades competentes de Migración Colombia con el fin de corroborar su estatus migratorio.

Es así, como se aprecian diferencias de acción final por parte de la institucionalidad colombiana, en razón del fundamento que valide o desestime el material probatorio recaudado en un caso de espionaje, dependiendo de ello la aplicabilidad del Art. 463 del Código Penal o la activación del protocolo de expulsión por parte de Migración Colombia.

\section{Conclusiones}

Partiendo de la fundamentación conceptual y la casuística expuesta, se evidencia que los instrumentos normativos vigentes y aplicables ante la cuestión del espionaje como elemento de riesgo para la Seguridad Nacional afrontan una serie de dificultades y contradicciones que impiden u obstaculizan la aplicación de medidas penales que castiguen con contundencia dicho tipo de actividades. No obstante, la adaptación de los requerimientos de protección a la Seguridad Nacional, en aras de identificar y disuadir la ejecutoria de casos de espionaje en territorio nacional, ha logrado cumplirse y proyectarse desde la figura de acción operacional de Migración Colombia, actuando esta dentro de los parámetros del Estado Social de Derecho vigente, que estipula el máximo grado garantista de respeto por la integridad de los sujetos detenidos por casos de espionaje, en concordancia con la línea de amparo de los derechos fundamentales. 
Por otra parte, en cuanto a los protocolos de acción a gestionarse ante la identificación de actividades de espionaje, efectuadas por agentes acreditados dentro de un cuerpo diplomático extranjero, opera el procedimiento consular de entendimiento entre la Cancillería colombiana y la respectiva embajada donde se localice el funcionario identificado como un elemento de riesgo contra la seguridad nacional, para así proceder a su respectiva expulsión del territorio nacional, en concordancia con los principios de inmunidad diplomática que ostenta ese tipo de actores. Es así como se ratifica la importancia por exponer ejes de revisión y reflexión, capaces de observar y discutir las capacidades domésticas para afrontar factores de riesgo y amenaza en lo concerniente al espionaje, y que en tanto sean capaces también de plasmar la aplicación de los principios normativos del DIH y tiendan a otorgar mayores herramientas jurídicas para la aplicación de medidas penales más contundentes. Para lo anterior, el acompañamiento de acciones interagenciales e interinstitucionales, de impacto en el procesamiento de información y material probatorio sobre actividades de espionaje, ha de ser uno de los puntos a fortalecer dentro de los hallazgos evidenciados en este texto; en paralelo, también se ha de instar por consumar trabajos de diseño institucional capaces de superar factores contraproducentes que emergen ante la confrontación de la amenaza del espionaje por parte de los organismos de seguridad del Estado, tal y como lo pueden ser eventuales recelos y rivalidades entre las agencias encargadas de recolectar esos datos.

Una de las discusiones derivadas de los hallazgos y el diagnóstico del diseño institucional colombiano es cómo garantizar que la orientación de procesos jurídicos y de regulación migratoria realizados sobre ciudadanos extranjeros combine el tradicional respeto por los principios del DIH y el requerimiento fundamental por garantizar la protección de información estratégica que pretenda ser sustraída por agentes extranjeros desde el territorio nacional. No obstante, la realidad vigente da cuenta de algunas falencias y debilidades para fortalecer el carácter penal de la medida violatoria de los intereses nacionales por parte de sujetos que ejecutan actividades de espionaje en Colombia; las formas diferenciales de abordaje institucional vigentes relacionan cómo la mayoría de los casos reportados acogen la figura discrecional de la expulsión por parte de Migración Colombia; no obstante, esto significa que los sujetos dispuestos bajo ese protocolo no son condenados bajo el marco legal vigente, ya sea por la insuficiencia de material probatorio alegada por el juez encargado del caso o por otras determinaciones argumentadas por la autoridad migratoria, facultada desde sus capacidades de Policía Judicial.

Por último, cabe resaltar que la condición de respeto por el DIH en Colombia legitima la autonomía nacional para proceder a judicializar acciones asociadas con el espionaje, dando cuenta de cómo la arquitectura del Estado Social de Derecho sí permite adelantar procesos en la materia, siempre y cuando se garantice el debido proceso y el principio de legalidad acorde con la estructura constitucional. No obstante, en función de plasmar un ejercicio normativo eficiente ante la disposición del Código Penal, la articulación informativa deseable para determinar elementos probatorios por espionaje, presenta notables limitaciones al ser sujeto de interpretación autónoma por parte de los jueces que manejen ese tipo de casos, con lo cual el camino viable para garantizar la prevalencia de la Seguridad Nacional como criterio de peso para aplicar medidas penales privativas de la libertad no está garantizado. Entre tanto, ello significa que seguirá dándose ese nivel de importancia a través de las medidas discrecionales de expulsión llevadas a cabo por Migración Colombia. 


\section{Referencias}

Collazos, M. (20 de mayo de 2019). rcnradio.com. Obtenido de https://www.rcnradio. com/judicial/ordenan-libertad-cubano-acusado-de-planear-atentados-en-bogota

ColombianRiskAnalisys.(2021).ElpapeldeColombiaenmediodelacompetenciadelasgrandes potencias. Colombia Risk Analysis. Obtenido de https://23aa0fcf-8783-47cb-a14474c17bafa633.usrfiles.com/ugd/23aa0f_33dc2226afc04cc08098ec6995bf6885.pdf

Congreso de la República. (17 de abril de 2013) Ley Estatutaria Nº 1621 [Ley 1621 de 2013].

Congreso de la República. (24 de julio del 2000). Por la cual se expide el Código Penal. [Ley 599 de 2000].

Constitución Política de Colombia [Const.] (1991). Artículos 152 y 153 [Titulo VI].

Corte Constitucional. (1995). Sentencia C-225/95.

Corte Constitucional. (2012). Sentencia C-540/12. [MP Jorge Iván Palacio Palacio].

Departamento Administrativo de la Función Pública. (31 de octubre de 2011). Por el cual se crea la Unidad Administrativa Especial Migración Colombia, se establece su objetivo y estructura. [Decreto 4062 de 2011].

Derecho internacional relativo a la conducción de las hostilidades (Comité internacional de la Cruz Roja 20 de junio de 2005). Obtenido de https://www.icrc.org/es/ publication/derecho-internacional-relativo-la-conduccion-de-las-hostilidadescoleccion-de-convenios

El Tiempo. (20 de diciembre de 2018). eltiempo.com. Obtenido de https://www.eltiempo. com/colombia/otras-ciudades/quien-es-carlos-manuel-pino-garcia-307260

El Tiempo. (19 de marzo de 2019). eltiempo.com. Obtenido de https://www.eltiempo. com/justicia/investigacion/aumenta-alerta-por-espionaje-a-bases-militarescolombianas-339668

Estatuto de combatiente y de prisionero de guerra (Comité internacional de la Cruz Roja, 28 de febrero de 1989). Obtenido de https://www.icrc.org/es/doc/ resources/documents/misc/5tdm 4 r.htm

IV. Convenio de Ginebra (Comité internacional de la Cruz Roja, 12 de agosto de 1949). Obtenido de https://www.icrc.org/es/doc/resources/documents/treaty/treatygc-4-5tdkyk.htm

Javeriana, P. U. (2002). Reflexiones en torno al Derecho Internacional Humanitario y 
el Nuevo Código Penal Colombiano. Bogotá: Pontificia Universidad Javeriana, Facultad de Ciencias Jurídicas, Departamento de Derecho Penal.

Jiménez, W. G., Angulo, L. L., Castiblanco, Y. P., Gómez, M. L., Rey, L. J., Solano, L. T., y Urquijo, Y. C. (2016). Ley estatutaria: ¿avance hacia la garantía del derecho fundamental a la salud? Revista Colombiana de Cirugía (31), 81-90. Obtenido de http://www.scielo.org.co/pdf/rcci/v31n2/v31n2a2.pdf

Lowenthal, M. (2000). Counterintelligence. En Intelligence: From secrets to policy (págs. 145-156). CQ Press.

Ministerio de Relaciones Exteriores. (26 de mayo de 2015). Por medio del cual se expide el Decreto Único Reglamentario del Sector Administrativo de Relaciones Exteriores. [Decreto 1067 de 2015].

Ministerio de Relaciones Exteriores. (2 de agosto de 2017). Por la cual se dictan disposiciones en materia de visas [...]. [Resolución 6045 de 2017].

Olaya, M. A. (2004). El bloque de constitucionalidad en la jurisprudencia de la Corte Constitucional colombiana. Precedente Revista Jurídica, 79-102.

Ramos, L. N. (1999). Policía Judicial. Composición, funciones y principios de actuación. Unidades orgánicas de la Policía Judicial: su dependencia funcional y orgánica. Eguzkilore: cuaderno del Instituto Vasco de Criminología, 117-156.

Sanín, G. T. (2018). Estado, política y economía en Colombia -capitalismo burocrático y gansteril-. Señal Editora. 